\title{
Assessment of plasma chitotriosidase activity, CCL18/PARC concentration and NP-C suspicion index in the diagnosis of Niemann-Pick disease type C: a prospective observational study
}

Isabel De Castro-Orós ${ }^{1,2^{*}+}$, Pilar Irún ${ }^{1,2,3+}$, Jorge Javier Cebolla ${ }^{1,4}$, Victor Rodriguez-Sureda ${ }^{3,5}$, Miguel Mallén ${ }^{1}$, María Jesús Pueyo ${ }^{1}$, Pilar Mozas ${ }^{1}$, Carmen Dominguez ${ }^{3,5}$, Miguel Pocoví $^{1,2}$

and on behalf of the Spanish NP-C Group

\begin{abstract}
Background: Niemann-Pick disease type C (NP-C) is a rare, autosomal recessive neurodegenerative disease caused by mutations in either the NPC1 or NPC2 genes. The diagnosis of NP-C remains challenging due to the non-specific, heterogeneous nature of signs/symptoms. This study assessed the utility of plasma chitotriosidase (ChT) and Chemokine (C-C motif) ligand 18 (CCL18)/pulmonary and activation-regulated chemokine (PARC) in conjunction with the NP-C suspicion index (NP-C SI) for guiding confirmatory laboratory testing in patients with suspected NP-C.

Methods: In a prospective observational cohort study, incorporating a retrospective determination of NP-C SI scores, two different diagnostic approaches were applied in two separate groups of unrelated patients from 51 Spanish medical centers ( $n=118$ in both groups). From Jan 2010 to Apr 2012 (Period 1), patients with $\geq 2$ clinical signs/ symptoms of NP-C were considered 'suspected NP-C' cases, and NPC1/NPC2 sequencing, plasma chitotriosidase (ChT), CCL18/PARC and sphingomyelinase levels were assessed. Based on findings in Period 1, plasma ChT and CCL18/ PARC, and NP-C SI prediction scores were determined in a second group of patients between May 2012 and Apr 2014 (Period 2), and NPC1 and NPC2 were sequenced only in those with elevated ChT and/or elevated CCL18/PARC and/ or NP-C SI $\geq 70$. Filipin staining and 7-ketocholesterol (7-KC) measurements were performed in all patients with NP-C gene mutations, where possible.

Results: In total across Periods 1 and 2, 10/236 (4\%) patients had a confirmed diagnosis o NP-C based on gene sequencing (5/118 [4.2\%] in each Period): all of these patients had two causal NPC1 mutations. Single mutant NPC1 alleles were detected in 8/236 (3\%) patients, overall. Positive filipin staining results comprised three classical and five variant biochemical phenotypes. No NPC2 mutations were detected. All patients with NPC1 mutations had high ChT activity, high CCL18/PARC concentrations and/or NP-C SI scores $\geq 70$. Plasma 7-KC was higher than control cut-off values in all patients with two NPC1 mutations, and in the majority of patients with single mutations. Family studies identified three further NP-C patients.
\end{abstract}

\footnotetext{
*Correspondence: isadco@gmail.com

${ }^{\dagger}$ Isabel De Castro-Orós and Pilar Irún should be regarded as joint first

authors

${ }^{1}$ Department of Biochemistry and Molecular and Cellular Biology, Faculty

of Science, University of Zaragoza, C. Pedro Cerbuna 12, 50009 Saragossa,

Spain

Full list of author information is available at the end of the article
} 
Conclusion: This approach may be very useful for laboratories that do not have mass spectrometry facilities and therefore, they cannot use other NP-C biomarkers for diagnosis.

Keywords: Niemann-Pick disease type C, Chitotriosidase, CCL18/PARC, NP-C suspicion index, 7-ketocholesterol, Diagnosis, Screening

\section{Background}

Niemann-Pick disease type C (NP-C) is a rare inherited lysosomal storage disorder with an estimated incidence of 1:120,000 live births [1]. Mutations in either of the two genes, NPC1 or NPC2, have been described as the cause of the disease [1]. Approximately $95 \%$ of patients with a genetic diagnosis have NPC1 mutations: NPC1 encodes a large membrane glycoprotein with mostly late-endosomal localization [2]. Other patients have mutations in the NPC2 gene, which encodes a small soluble lysosomal protein that binds cholesterol with high affinity $[1,3]$.

The diagnosis of NP-C remains challenging as neurological signs of the disease are extremely varied in terms of severity and age at onset $[1,4]$. In particular, the age at onset of neurological manifestations has a major influence on disease progression and prognosis, and patients can be categorized on the basis of early-infantile, lateinfantile, juvenile and adolescent/adult neurological onset to aid clinical management and family counselling [4]. Generally, patients with very early-onset disease are detected and diagnosed based on pronounced visceral symptoms such as prolonged neonatal jaundice, fetal hydrops and/or ascites [1, 5]. Diagnoses in later-onset patients depend more on the recognition of typical neurological signs such as vertical supranuclear gaze palsy (VSGP), developmental delay, cerebellar ataxia, and gelastic cataplexy, which may or may not be detected alongside splenomegaly. However, visceral symptoms can appear long before neurological signs and often go unrecognized, particularly in those with adolescent/adultonset disease [1, 4].

The NP-C suspicion index (NP-C SI) was developed by an international team of experts for the detection of NP-C among patients suspected of having the disease, and is based on easily assessed patient clinical symptoms and medical history [6]. Patients scoring $\geq 70$ on the NP-C SI should be considered as possibly having NP-C and should undergo further, specific laboratory tests. Scores $<40$ on the NP-C SI indicate a low likelihood of NP-C [4].

Niemann-Pick disease type $C$ diagnoses can only be confirmed using specific laboratory tests [4]. Filipin staining in patient skin fibroblast cultures is a sensitive and specific test to identify impaired intracellular cholesterol transport and homeostasis. This test has been considered the gold standard method for diagnosing NP-C because it establishes the biochemical phenotype of the disease and provides useful functional evidence of the pathogenicity of novel gene mutations [4, 7-10]. However, recent progress in gene mutation analysis, the lack of correlation between some causal mutations and 'variant' filipin staining patterns, and the fact that the filipin test is time consuming and expensive, have led to this gold standard being challenged $[8,11]$. Most patients with NP-C (80-85\%) show a 'classical' pattern of cholesterol storage featuring numerous, strongly fluorescent perinuclear vesicles. However, some patients display a 'variant' biochemical phenotype that features a less distinct, more variable pattern $[9,10]$.

The establishment of reliable biomarkers for the presence and progression of NP-C represents an important goal. Chitotriosidase (ChT) is a human plasma chitinase enzyme that shows markedly elevated activity in a variety of lysosomal storage disorders [12, 13], High plasma levels reflect gradual intralysosomal accumulation of the enzyme in lipid-loaded macrophages, which secrete it [14]. Plasma ChT is widely used in Gaucher disease (GD) to monitor treatment response to enzyme replacement therapy (ERT) [12]. Ries et al. reported that patients with NP-C showed a mean plasma ChT activity of $856 \pm 721 \mathrm{nmol} / \mathrm{mL}$ *h compared with $55 \pm 35.6 \mathrm{nmol} /$ $\mathrm{mL}$ *h in individuals with miscellaneous other diseases, and $13,761 \pm 10,348 \mathrm{nmol} / \mathrm{mL}$ "h in patients with GD [15]. A ChT activity $>200 \mathrm{nmol} / \mathrm{mL}^{*} \mathrm{~h}$ is considered pathological [15]. However, 3-6\% of individuals with European ancestry have the c.1049_1072dup24 polymorphism of the ChT gene (CHIT1), which leads to a complete lack of ChT activity $[16,17]$.

Chemokine (C-C motif) ligand 18 (CCL18)/pulmonary and activation-regulated chemokine (PARC) is produced mainly by monocytes/macrophages that constitutively express it only at low levels in normal circumstances, but its production can be up-regulated in these cells by macrophage activators [18]. Plasma CCL18 levels have been reported to increase 29 -fold in symptomatic GD patients [19] leading to its use as a further disease biomarker [20]. Pineda et al. observed that patients with early- or lateinfantile onset NP-C had higher plasma CCL18/PARC activities compared with juvenile-onset or asymptomatic patients, and proposed CCL18/PARC as an alternative marker in NP-C patients with ChT deficiency [20,21].

Levels of several cholesterol oxidation products (oxysterols) have recently been proposed as sensitive and 
specific markers for NP-C screening and/or diagnosis. Increased plasma 7-ketocholesterol (7-KC) levels have been reported in NP-C patients and in an NP-C mouse model [7, 22]. Further, Porter et al. have described an oxysterol profile specific for NP-C that correlates with the age of disease onset as well as disease severity [23].

In the following study, we evaluated whether plasma ChT activity and CCL18/PARC concentration might complement clinical assessments for the identification of patients who should undergo further, specific testing for NP-C. We also compared these biomarker analyses with NP-C SI scores and with findings from plasma oxysterol measurements.

\section{Methods}

\section{Patients and study design}

Patients from 51 Spanish medical centres specializing in neurodegenerative disorders who had two or more symptoms typically seen in NP-C were considered as possibly having the disease, and were enrolled into this prospective observational study combined with a retrospective determination of NP-C SI scores following reference to a questionnaire with a list of symptoms and clinical data of interest (see Additional file 1). Patients presenting with at least two of the following symptoms were included in the study: bipolar disorder; schizophrenia; depression; one other clinical symptom. Medical chart data relating to demographics, diagnosis, and recorded disease characteristics were obtained from referring physicians.

The study comprised two observation periods, during which different diagnostic approaches were followed. In Period 1, which ran from January 2010 to April 2012, patients with two or more clinical NP-C signs and symptoms were considered as having 'suspected NP-C'. NPC1/NPC2 sequencing, ChT and sphingomyelinase activities, and CCL18/PARC concentration were assessed in all patients. If either one or two NPC1 or NPC2 mutations were identified, filipin staining and oxysterol measurements were also performed, where possible. In Period 2, which ran from May 2012 to April 2014, ChT activity, CCL18/PARC concentration and NP-C SI scores were analyzed in all patients, and NPC1/NPC2 sequencing was performed only in those who had elevated ChT and CCL18/PARC values (greater than mean + two standard deviations [SD] versus control values), and/or an NP-C SI score $\geq 70$. Filipin staining and/or plasma oxysterol measurements were performed, where possible, in all patients with either one or two NPC1 or NPC2 mutations.

\section{Genetic analysis}

DNA was isolated from EDTA blood samples using standard methods. The promoter and 25 exons of NPC1, and respective exon-intron boundaries, were amplified simultaneously in 24 PCR reactions using oligonucleotide primers developed in-house at the University of Zaragoza. The promoter, coding regions of the five exons of NPC2 and respective exon-intron boundaries were amplified in a single multiplex reaction with linkertailed primers. Amplification products were joined into one DNA fragment using universal external primers, and the resulting amplicons were purified using the Illustra ${ }^{\mathrm{TM}}$ ExoStar $^{\mathrm{TM}} 1$-Step system (GE Healthcare, UK), followed by $5^{\prime}$ to $3^{\prime}$ sequencing in an ABI 3500XL DNA analyzer (Applied Biosystems, USA).

To analyze splicing variants, total RNA was isolated from cultured fibroblasts $\left(1 \times 10^{6}\right.$ cells/column $)$ using the RNeasy Mini Kit (Qiagen, USA), and genomic DNA was removed using the RNase-Free DNase Set (Qiagen). RNA (200 ng for each reaction) was used for cDNA synthesis with random hexamer primers using RevertAid $\mathrm{H}$ (Qiagen) minus first strand cDNA synthesis. Two fragments, one from c.227 to c.976 and another from c.1793 to c.2359, were amplified by PCR, purified using the Illustra $^{\mathrm{TM}}$ ExoStar $^{\mathrm{TM}} 1$-Step system (GE Healthcare), followed by $5^{\prime}$ to $3^{\prime}$ sequencing in an ABI 3500XL DNA analyzer (Applied Biosystems). All primer sequences used for PCR are available upon request.

Patients with only one NPC1 mutation were further analyzed by Multiplex Ligation-dependent Probe Amplification $\left(\right.$ SALSA $^{\circledR}$ MLPA $^{\circledR}$ P193 NPC1 version A2; MRC Holland, Netherlands) in order to find any rearrangements, with data normalized versus three healthy controls.

A number of software databases (PolyPhen-2 [24], SIFT [25] and MutationTaster [26]) were used to evaluate the pathogenicity of newly identified genetic variants that implied non-synonymous changes. The effect of variants in potential splicing sites was predicted using NetGene2 and NNSplice by analyzing the structure of donor and acceptor sites with a separate neural network recognizer for each site [27, 28]. Common polymorphisms were excluded, and gene variations were compared with databases of the National Center for Biotechnology Information (NCBI; http://www.ncbi.nlm.nih.gov/), Ensembl (http://www.ensembl.org/), the 1000 Genomes project (http://www.1000genomes.org/), and the Exome Variant Server (http://evs.gs.washington.edu/EVS/). All mutations were described according to the latest Human Genome Variation Society (HGVS) recommendations (http://www.hgvs.org/mutnomen).

\section{Chitotriosidase activity and CCL18/PARC concentration}

Plasma for biomarker measurements was separated from EDTA blood samples. Plasma ChT activity was measured 
using the fluorogenic substrate 4MU-chitotrioside (Sigma Chemical Co., USA), as described previously [12]. Genotyping for the 24-bp insertion in exon 10 of the CHIT1 gene (c.1049_1072dup24) was performed as described by Irun et al. [16]. Given that ChT activity is roughly half of normal or zero in patients who are heterozygous and homozygous for the c.1049_1072dup24 CHIT1 mutation, respectively, ChT levels were multiplied by two in heterozygous patients. The concentration of the chemokine CCL18/PARC was analyzed by enzyme-linked immunosorbent assay (ELISA; R\&D Systems Europe, Ltd, UK) as described elsewhere [19]. Mean \pm SD control values determined in 36 patients who did not have lysosomal disorders were: $46.1 \pm 30.2 \mathrm{nmol} / \mathrm{mL} / \mathrm{h}$ for ChT activity, and $52.5 \pm 30.3 \mathrm{ng} / \mathrm{mL}$ for CCL18/PARC concentration.

\section{NP-C suspicion index}

Individuals $>4$ years old were assessed using the NP-C SI as described in detail elsewhere (http://www.npc-si. com) [6]. The NP-C SI was published in 2012, but it was considered useful in the context of the current study and was therefore applied retrospectively in Period 1 and prospectively in Period 2.

\section{Filipin staining}

Normal and NP-C fibroblasts were cultured using standard laboratory methods, but with some adaptations (see Additional file 2). Only homogeneous confluent cell monolayers grown over 3-6 passages, and covering an area of $25 \mathrm{~cm}^{2}$, were studied. Fluorescent staining of lysosomal cholesterol in fibroblast cultures was performed based on a well-known cytochemical method in low-density lipoprotein (LDL)-challenged cells, and perinuclear cholesterol accumulation was assessed as described previously [9].

\section{Oxysterol analysis}

The concentration of 7-KC was measured in all patients with available plasma samples and at least one identified NPC1 mutation. Extraction of 7-KC from plasma samples was conducted according to the method described by Lin et al. [22], and 7-KC was quantified using liquid chromatography-tandem mass spectrometry (LC-MS/ MS) based on a slightly modified version of the method described by Baila-Rueda et al. [29]. Briefly, a calibration curve for 7-KC showed a correlation coefficient of 0.995 , with an assay linear range of $2-800 \mathrm{ng} / \mathrm{mL}$. The lower limits of detection (LOD) and quantitation were 1 and $2 \mathrm{ng} / \mathrm{mL}$, respectively. Intra-day and inter-day variations were $<5$ and $<11 \%$, respectively. Mean \pm SD control values determined in 36 patients who did not have lysosomal disorders were $15.99 \pm 14.67 \mathrm{ng} / \mathrm{mL}$.

\section{Family studies}

Genetic analyses were applied in all available first-degree relatives of index cases possessing two $N P-C$ gene mutations in order to establish whether mutations were located in the same allele or in different alleles, and to identify other NP-C patients.

\section{Statistical methods}

As this was an observational study, data analyses were descriptive in nature.

\section{Results \\ Patients and diagnoses}

In total, 236 unrelated patients with suspected NP-C were included in Periods 1 and 2 (Fig. 1). All 118 patients included during Period 1 underwent NPC1/NPC2 sequencing, among whom five patients were found to be homozygous or compound heterozygotes for NPC1 mutations (Table 1), and three were found to be carriers of single heterozygous NPC1 mutations (Table 2).

In Period 2, 118 patients were assessed for biomarkers (ChT and CCL18/PARC). But based on biomarkers results, only 43 out of 118 patients in Period 2 underwent genetic testing. Five of the 118 patients enrolled during Period 2 were homozygous or compound heterozygotes for NPC1 mutations (Table 1), and a further five were carriers of one NPC1 allele variant (Table 2). Sphingomyelinase deficiency was detected in two patients who were subsequently diagnosed with Niemann-Pick disease type B based on SMPD1 gene mutation analysis. Another patient showed acid glucosidase deficiency (with $<10 \%$ of normal activity), and was diagnosed with GD after identification of two $G B A$ mutations.

\section{$\mathrm{ChT}$ and CCL18/PARC analyses}

The mean \pm SD plasma CCL18/PARC concentration and ChT activity in the five patients from Period 1 with an identified genetic cause for NP-C were $289 \pm 286 \mathrm{ng} / \mathrm{mL}$ (range $88-788 \mathrm{ng} / \mathrm{mL}$ ) and $380 \pm 374 \mathrm{nmol} / \mathrm{mL}^{*} \mathrm{~h}$ (range 156-1045 nmol/mL"h), respectively. Patients with one mutation had substantially lower values: $185 \pm 56.3 \mathrm{ng} /$ $\mathrm{mL}$ (range $123-233 \mathrm{ng} / \mathrm{mL}$ ) and $110 \pm 56.1 \mathrm{nmol} / \mathrm{mL} * \mathrm{~h}$ (range 53-167 nmol/mL*h), respectively. Excluding patient NPC4B who had no available data, all patients with NPC1 mutations showed CCL18/PARC concentrations and/or corrected ChT activities that were at least two SDs greater than control values.

In Period 2, 43/118 (36\%) patients had CCL18/PARC concentrations and/or corrected ChT activities at least two SDs greater than control values (i.e., CCL18/ PARC $>115 \mathrm{ng} / \mathrm{mL}$ and/or ChT $>150 \mathrm{nmol} / \mathrm{mL}$ h) [16], or normal biomarker levels but an NP-C SI score $>70$. In 


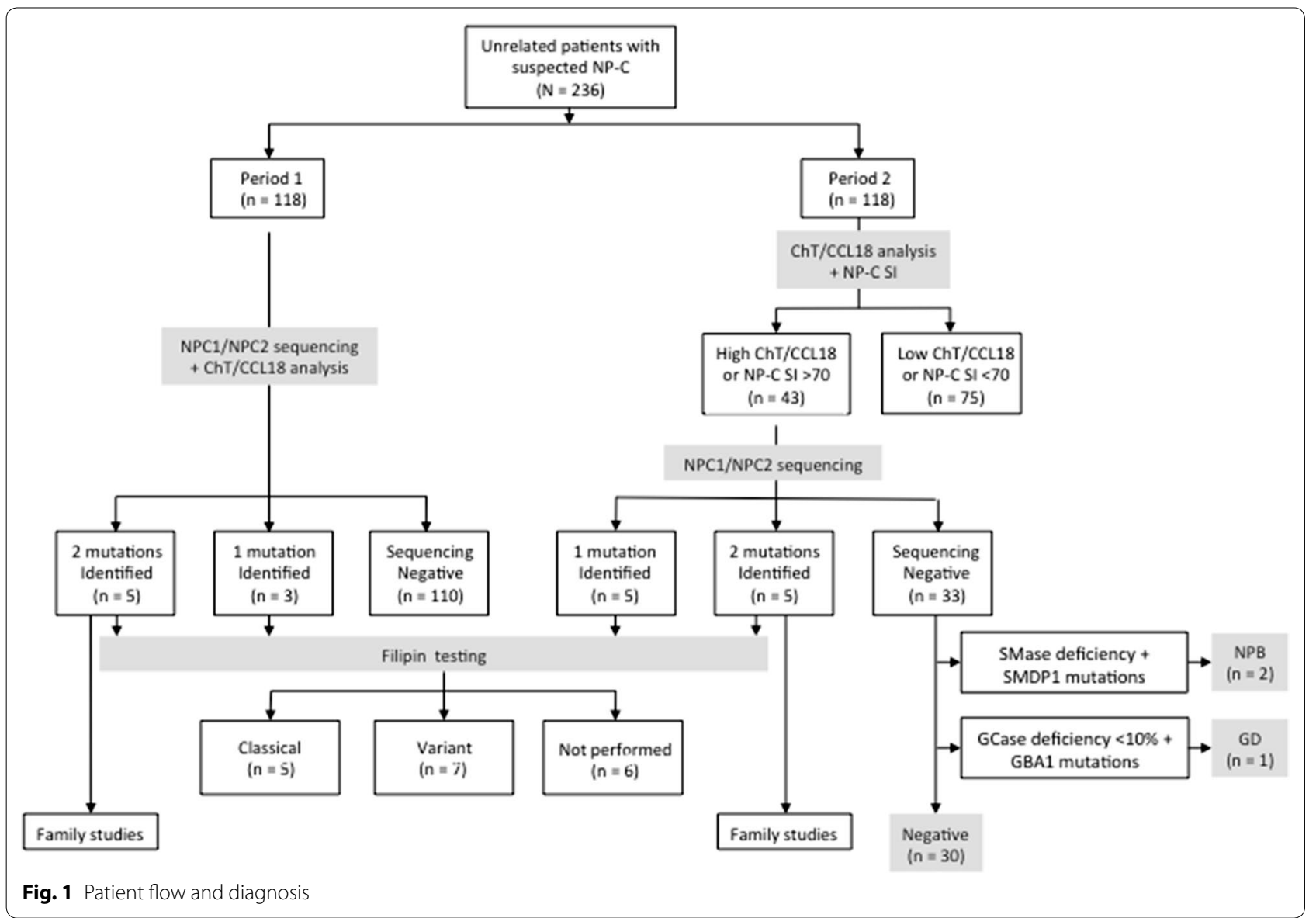

this Period, 18 patients showed elevated levels of both ChT and CCL18/PARC, six only showed elevated ChT activity, and 14 had elevated CCL18/PARC concentrations. Five patients had only NP-C SI $\geq 70$, and $13 / 118$ subjects from Period 2 had NP-C SI $\geq 70$. NPC1 and $N P C 2$ mutation analyses were therefore conducted in 43 patients in this group.

Analysis for the c.(1049_1072) dup24 CHIT1 genotype identified four and three homozygous patients in Periods 1 and 2, respectively, and five and 10 heterozygous patients in Periods 1 and 2, respectively. The homozygous patients were not excluded from the study because it was still possible to analyze CCL18/PARC concentration and NP-C SI scores.

\section{NP-C suspicion index analysis}

Overall, 79 (33\%) of all enrolled patients had an NP-C SI risk prediction score $\geq 70$ following clinical screening, $17(7.1 \%)$ of whom were children $<4$ years old. Six out of 10 of the NP-C patients who had two NPC1 mutations had a high suspicion of NP-C (risk prediction score $\geq 70$ ) (Table 1 ). The remaining patients were either $<4$ years old or had an NP-C SI $<70$. Among patients in the
single-NPC1 mutation group, of which four were children aged $<4$ years, only one patient had an NP-C SI $\geq 70$ (Table 2).

\section{Filipin staining}

Skin biopsies were performed in all patients who consented to them, and diagnoses of NP-C were confirmed based on abnormal filipin staining in these cases (Table 1). Six patients with a single NPC1 mutation showed a variant filipin staining pattern, while two showed a classical staining pattern (Table 2).

\section{Oxysterol analysis}

Plasma 7-KC concentrations were analyzed in $17 \mathrm{NP}-\mathrm{C}$ patients and 21 relatives. The mean \pm SD 7 -KC level among all patients with two NPC1 mutations was $350.8 \pm 221.8 \mathrm{ng} / \mathrm{mL}$ (range $103-761 \mathrm{ng} / \mathrm{mL}$ ), and all of these patients had plasma 7-KC concentrations higher than the optimal control cut-off value $(102.8 \mathrm{ng} / \mathrm{mL})$ in our laboratory (Table 3). Among patients with one mutation, the mean \pm SD 7-KC concentration was also raised $(194 \pm 265.9 \mathrm{ng} / \mathrm{mL}$ [range $<2-761 \mathrm{ng} / \mathrm{mL}])$, although only $4 / 7$ of these patients, all of whom had positive 
Table 1 Mutational and biochemical features of NP-C patients with two NPC1 mutations

\begin{tabular}{|c|c|c|c|c|c|c|c|c|c|c|}
\hline Patient ID & $\begin{array}{l}\text { Variant } \\
\text { allele } 1 \\
\text { amino acid }\end{array}$ & $\begin{array}{l}\text { Variant } \\
\text { allele } 1 \\
\text { reference }\end{array}$ & $\begin{array}{l}\text { Variant } \\
\text { allele } 2 \\
\text { amino acid }\end{array}$ & $\begin{array}{l}\text { Variant } \\
\text { allele } 2 \\
\text { reference }\end{array}$ & $\begin{array}{l}\text { CCL18/ } \\
\text { PARC } \\
\text { ng/mL }\end{array}$ & $\begin{array}{l}\text { ChT(dup24) } \\
\text { nmol/mL.h }\end{array}$ & $\begin{array}{l}\text { NP-C SI } \\
\text { score }\end{array}$ & $\begin{array}{l}\text { Filipin } \\
\text { staining }\end{array}$ & $\begin{array}{l}\text { 7-KC } \\
\mathrm{ng} / \mathrm{mL}\end{array}$ & Clinical form \\
\hline \multicolumn{11}{|l|}{ Period 1} \\
\hline NPC1A & p.(GIn775Pro) & {$[32]$} & p.(Asp1097Asn) & [31] & 156 & 188 (Het) & 145 & ND & 260 & Adult \\
\hline $\mathrm{NPC2A}$ & p.(Arg1059*) & [30] & p. $\left(\operatorname{Arg} 1059^{*}\right)$ & {$[30]$} & 788 & 1045 (Neg) & $N C(<4$ y.o. $)$ & Classical & 650 & Early infantile \\
\hline NPC3A & p.(Pro1007Ala) & {$[36]$} & p.(Asn222Ser) & [34] & 88 & 266 (Neg) & 60 & Variant & 103 & Adult \\
\hline NPC4A & p.(Arg518Trp) & {$[35]$} & p.(Gly992Trp) & {$[36]$} & 151 & 244 (Neg) & 195 & ND & 213 & Adult \\
\hline NPC5A & p.(Trp942Cys) & [30] & p.(Arg1173Gly) & New & 266 & 156 (Het) & 95 & ND & 178 & Adult \\
\hline \multicolumn{11}{|l|}{ Period 2} \\
\hline NPC6A & p.(Arg372Trp) & {$[30]$} & p.(Thr1036Met) & [2] & 466 & 415 (Neg) & 20 & ND & 238 & Adult \\
\hline NPC7A & p.(Glu1188 fs*54) & {$[30]$} & p.(Thr375Ala) & New & 265 & 75 (Neg) & 200 & ND & 398 & Adult \\
\hline NPC8A & p.(lle1061Thr) & {$[17]$} & p.(Ile1061Thr) & [17] & 1137 & 1477 (Neg) & NC (<4 y.o.) & ND & 514 & Early infantile \\
\hline NPC9A & p.(Cys177Tyr) & {$[35]$} & p.(Val664Met) & [34] & 516 & 614 (Neg) & 190 & Classical & 193 & Adult \\
\hline NPC10A & p.(Leu107Cfs*5) & [32] & $\begin{array}{l}\text { p.(Glu61 +? } \\
\quad \text { Asp211 + ?)dup }\end{array}$ & New & 1048 & 812 (Het) & NC (<4 y.o.) & Classical & 761 & Early infantile \\
\hline
\end{tabular}

Mutations were described according to the latest HGVS recommendations (http://www.hgvs.org/mutnomen); ChT chitotriosidase (ChT activity of heterozygous individuals [dup24] was multiplied by two). NC not conducted (patient < 4 years old), ND no data available, Neg negative, 7-KC 7-ketocholesterol, LOD limit of detection $(=2 \mathrm{ng} / \mathrm{mL})$

Table 2 Mutational and biochemical features of 'NP-C uncertain' patients with only one NPC1 mutation

\begin{tabular}{|c|c|c|c|c|c|c|c|c|c|}
\hline Patient ID & $\begin{array}{l}\text { Variant allele } 1 \\
\text { amino acid }\end{array}$ & $\begin{array}{l}\text { Variant allele } 1 \\
\text { reference }\end{array}$ & $\begin{array}{l}\text { CCL18/PARC } \\
\mathrm{ng} / \mathrm{mL}\end{array}$ & $\begin{array}{l}\text { ChT (dup24) } \\
\text { nmol/mL.h }\end{array}$ & NP-C SI score & Filipin staining & MLPA & $\begin{array}{l}\text { 7-KC } \\
\mathrm{ng} / \mathrm{mL}\end{array}$ & Clinical form \\
\hline \multicolumn{10}{|l|}{ Period 1} \\
\hline $\mathrm{NPC1B}$ & p.(Leu846Pro) & New & 233 & 109 (Neg) & 55 & Variant & Negative & 134 & Adult \\
\hline $\mathrm{NPC2B}$ & p.(Phe1221Sfs*20) & {$[30]$} & 123 & 53 (Neg) & 60 & Classical & Negative & 34 & Adult \\
\hline NPC3B & p.(GIn775Pro) & [32] & 198 & 167 (Neg) & 55 & Classical & Negative & $<2$ & Adult \\
\hline \multicolumn{10}{|l|}{ Period 2} \\
\hline NPC4B & p.(Arg1274Trp) & New & ND & ND & NC (<4 y.o.) & Variant & Negative & ND & Early infantile \\
\hline NPC5B & p.(Glu451Lys) & [36] & 550 & 771 (Neg) & NC (<4 y.o.) & Variant & Negative & 258 & Early infantile \\
\hline NPC6B & p.(Asn222Ser) & {$[34]$} & 138 & 59 (Neg) & NC (<4 y.o. $)$ & Variant & Negative & 150 & Late infantile \\
\hline NPC7B & p.(GIn775Pro) & New & 416 & 132 (Neg) & 101 & Variant & Negative & 125 & Adult \\
\hline NPC8B & p.(Gln775Pro) & [32] & 39 & 24 (Neg) & 120 & Variant & Negative & 19 & Juvenile \\
\hline
\end{tabular}

Mutations were described according to the latest HGVS recommendations (http://www.hgvs.org/mutnomen); ChT chitotriosidase (ChT activity of heterozygous individuals [dup24] multiplied by two), NC not conducted (patient $<4$ years old), ND no data available, Neg negative, 7-KC 7-ketocholesterol, LOD limit of detection $(=2 \mathrm{ng} / \mathrm{mL})$

classical or variant filipin staining results, had plasma 7-KC concentrations higher than the cut-off value.

\section{Clinical manifestations}

Clinical manifestations are summarized in Table 4. The most prevalent manifestations in patients with a confirmed diagnosis of NP-C were neurological signs, including: ataxia, VSGP, dementia, dystonia and/or dysarthria. Psychiatric signs were the second most frequent class of manifestation, and included pre-senile cognitive decline/dementia, psychotic symptoms, and depression/ bipolar disorders. Unexplained splenomegaly with or without concurrent hepatomegaly were present in $5 / 10$ patients $(50 \%)$ with a confirmed diagnosis.

\section{NPC1 mutations}

Patients diagnosed with NP-C in this cohort showed wide heterogeneity of NPC1 variants. In total, eight new NPC1 variants were detected: four missense (p.(Thr375Ala), p.(Leu846Pro), p.(Arg1173Gly), p.(Arg1274Trp)), two different tentative splicing variants at intron 12 (c. $(1947+10 G>A)$ ) and exon 5 (c. $(612 \mathrm{C}>\mathrm{T})$, p.(Thr204Thr)), one deletion (c.318_318delC) that produces a frameshift change, p.(L107CfsX5), and one rearrangement (c. $(280+$ ? _c.630 + ?) dup (p.Glu61 + _Asp211 + ?) dup)) that leads to a duplication of exons 4 and 5 . In silico analysis with Polyphen2, SIFT and MutationTaster software indicated that these changes could affect protein function (Table 5). We also found 17 rare NPC1 variants previously 
Table 3 Patient demographics and diagnostic features among all study patients

\begin{tabular}{|c|c|c|c|}
\hline & $\begin{array}{l}\text { NP-C positive } \\
\text { (two NPC1 } \\
\text { mutations) } \\
(n=10)\end{array}$ & $\begin{array}{l}\text { NP-C uncertain } \\
\text { (one NPC1 } \\
\text { mutation) } \\
(\mathrm{n}=8)\end{array}$ & $\begin{array}{l}\text { NP-C } \\
\text { negative } \\
(n=218)\end{array}$ \\
\hline \multicolumn{4}{|l|}{ Age (years) } \\
\hline Mean \pm SD & $28 \pm 21$ & $28 \pm 28$ & $44 \pm 22$ \\
\hline Median (range) & $35(1.4-62)$ & $21(0.8-68)$ & $46(0.03-83)$ \\
\hline \multicolumn{4}{|l|}{ Gender (n) } \\
\hline Male & 4 & 6 & 126 \\
\hline Female & 6 & 2 & 92 \\
\hline \multicolumn{4}{|l|}{ NP-CSI (points) } \\
\hline Mean \pm SD & $129 \pm 72.1$ & $78.2 \pm 27.1$ & $58.9 \pm 39.2$ \\
\hline Median (range) & 145 (20-200) & $69.1(55-120)$ & $55(5-245)$ \\
\hline \multicolumn{4}{|l|}{$\begin{array}{l}\text { ChT activity } \\
\text { (nmol/mL/h) }\end{array}$} \\
\hline Mean \pm SD & $553 \pm 479$ & $187 \pm 262$ & $122 \pm 423$ \\
\hline Median (range) & $255(75-1477)$ & $109(24-771)$ & $53(11-5149)$ \\
\hline \multicolumn{4}{|c|}{ CCL18 conc. $(\mathrm{ng} / \mathrm{mL})$} \\
\hline Mean \pm SD & $481 \pm 370$ & $242 \pm 179$ & $95 \pm 178$ \\
\hline Median (range) & $266(88-1137)$ & $198(39-550)$ & $(11-1513)$ \\
\hline \multicolumn{4}{|l|}{$\begin{array}{l}7-K C \\
(n g / m L)\end{array}$} \\
\hline Mean \pm SD & $351 \pm 222$ & $103 \pm 91.20$ & ND \\
\hline Median (range) & $249(103-761)$ & $198(<2-761)$ & \\
\hline \multicolumn{4}{|l|}{ Filipin staining (n) } \\
\hline Classical & 3 & 2 & ND \\
\hline Variant & 1 & 6 & \\
\hline No data & 6 & 0 & \\
\hline
\end{tabular}

All patients, including those from both Periods 1 and 2. Data expressed as mean $\pm S D$, median and range (minimum-maximum). Normal biomarker values calculated in 36 patients without lysosomal disorders were: $46.1 \pm 30.2 \mathrm{nmol} /$ $\mathrm{mL} / \mathrm{h}$ for ChT activity; $52.5 \pm 30.3 \mathrm{ng} / \mathrm{mL}$ for CCL18/PARC concentration; and $15.99 \pm 14.67 \mathrm{ng} / \mathrm{mL}$ for $7-\mathrm{KC}$ concentration. $N D$ no data available

associated with NP-C. A further 17 common NPC1 variants or polymorphisms were also observed, including: c. $(-22 \mathrm{~A}>$ C), p.(Tyr129Tyr), p.(His215Arg), p.(Pro237Ser), p.(Ser322Ser), p.(Asn490Thr), p.(Met642Ile), p.(Ile858Val), p.(Asn931Lys), c. $(1947+10 \mathrm{G}>\mathrm{C})$, c. $(1947+14 \mathrm{G}>\mathrm{T})$, c. $(2086+8 \mathrm{G}>\mathrm{C})$, c. $(2911+28 \mathrm{~T}>\mathrm{C})$, c. $(3246+46 \mathrm{C}>\mathrm{T})$, c. $(3591+35 \mathrm{C}>\mathrm{T})$, c. $(3754+34 \mathrm{~A}>\mathrm{G})$, and p.(Arg1266Gln). Five NPC2 polymorphisms were detected (p.(Gly52Gly), p.(Ser67Pro), p.(Pro86Leu), p.(Ser121Ala) and c. $(441+437 \mathrm{~T}>\mathrm{C}))$, but no allele mutations were identified.

Two variants of uncertain significance were detected (c. $(1947+10 \mathrm{G}>\mathrm{A})$ and c. $(612 \mathrm{C}>\mathrm{T}))$. Complementary DNA sequencing was conducted in fibroblast DNA obtained from patient NPC10 to better classify these two variants, and confirmed the presence of heterozygous mutations (p.(Leu107CysfsX5) and c. $(612 \mathrm{C}>\mathrm{T})$ [p.Thr204Thr]). We also identified the c. $(280+$ ? _c. $630+$ ? $)$ dup variant. The c. $(1947+10 \mathrm{G}>\mathrm{A})$ mutation was analyzed, and a splicing effect has not been observed.

\section{Family studies}

Forty-nine relatives were available for genetic analysis, and 22 were identified as carriers of at least one NPC1 mutation. Three relatives were identified as compound heterozygotes for NPC1 mutations: p.(Pro1007Ala)p.(Asn222Ser); p.(Trp942Cys)-p.(Arg1173Gly); and p.(Glu1188 fs*54)-p.(Thr375Ala). Further examination of these three individuals revealed clinical symptoms of $\mathrm{NP}-\mathrm{C}$, and they were subsequently diagnosed with NP-C.

\section{Discussion}

Due to the variability of age at onset and presentation in NP-C it is crucial to determine which diagnostic strategy might best help identify affected patients among suspected cases. We evaluated two different diagnostic pathways incorporating two well characterized biomarkers that have previously been used to monitor lysosomal storage disease progression (ChT activity and CCL18/ PARC concentration) alongside appraisals of clinical symptoms and NP-C SI assessments.

The diagnostic approach in Period 1 was to sequence all exon and exon-intron boundaries of $N P C 1$ and $N P C 2$ in patients with suspected NP-C based on the presence of two or more typical signs/symptoms. All patients with two NPC1 mutations, or a single NPC1 mutation plus a positive filipin test, had elevated ChT activity and/or CCL18/PARC concentration. Ninety-eight patients without NPC1 mutations did not show elevated ChT activity or CCL18/PARC concentration, but retrospective NP-C SI assessments showed that 42 of these patients had NP-C SI scores $\geq 70$. Based on findings from Period 1 , during period 2 we conducted $N P C 1$ and $N P C 2$ sequencing only in patients with elevated CCL18/PARC concentration and/or ChT activity, or with an NP-C SI $\geq 70$ for all individuals $>4$ years old. During this second period, five patients with homozygous or compound-heterozygous NPC1 mutations were successfully identified. Five carrier heterozygote patients with single $N P C 1$ variants and positive filipin test findings (classical or variant biochemical phenotypes) were also detected.

We observed the same NP-C detection rate $(5 / 118$; $4.2 \%$ ) using the criteria of study Period 1 (i.e., $N P C 1$ and $N P C 2$ sequencing in all cases of suspected NP-C) as we did using the criteria of study Period 2 [i.e., NPC1 and NPC2 sequencing only when the ChT activity and/or CCL18/PARC concentration were elevated and/or NP-C SI was $\geq 70 ; 5 / 118$ cases $(4.2 \%)$ ]. These findings suggest that the measurement of one or both of plasma ChT activity and CCL18 concentration in conjunction with 
Table 4 Clinical disease characteristics of all patients

\begin{tabular}{lllc}
\hline & $\begin{array}{l}\text { NP-C positive } \\
\text { (two NPC1 mutations) } \\
(\mathbf{n = 1 0 )}\end{array}$ & $\begin{array}{l}\text { NP-C uncertain } \\
\text { (one NPC1 mutation) } \\
(\mathbf{n = 8})\end{array}$ & $\begin{array}{c}\text { NP-C negative } \\
(\mathbf{n}=\mathbf{2 1 8})\end{array}$ \\
\hline $\begin{array}{l}\text { Neurological symptoms, } n \text { (\%) } \\
\text { Vertical supranuclear gaze palsy (VSGP) }\end{array}$ & $3(30 \%)$ & $3(37.5 \%)$ & $58(26.6 \%)$ \\
Gelastic cataplexy & $0(0 \%)$ & $0(0 \%)$ & $7(3.2 \%)$ \\
Ataxia, clumsiness or frequent falls & $5(50 \%)$ & $3(37.5 \%)$ & $89(40.8 \%)$ \\
Dysarthria and/or dysphagia & $2(20 \%)$ & $3(37.5 \%)$ & $31((14.2 \%)$ \\
Dystonia & $1(10 \%)$ & $1(12.5 \%)$ & $55(25.2 \%)$ \\
Psychiatric symptoms present, $n$ (\%) & & $1(12.5 \%)$ & $76(34.9 \%)$ \\
Pre-senile cognitive decline or dementia & $2(20 \%)$ & $0(0 \%)$ & $19(8.7 \%)$ \\
Psychotic symptoms (schizophrenia) & $1(10 \%)$ & $2(25 \%)$ & $49(22.5 \%)$ \\
Depression & $3(30 \%)$ & $1(12.5 \%)$ & $12(5.5 \%)$ \\
Bipolar disorders & $1(10 \%)$ & & $12(5.5 \%)$ \\
Visceral symptoms, $n$ (\%) & & $2(25 \%)$ & $15(6.9 \%)$ \\
Unexplained neonatal jaundice or cholestasis & $1(10 \%)$ & $3(37.5 \%)$ & $1(0.5 \%)$ \\
Unexplained splenomegaly \pm hepatomegaly & $6(60 \%)$ & $0(0 \%)$ & \\
Hydrops fetalis or fetal ascites & $0(0 \%)$ & & \\
\hline
\end{tabular}

Including those from both Periods 1 and 2

Table 5 In silico mutation predictions

\begin{tabular}{llll}
\hline Mutation & PolyPhen-2 & SIFT & Mutation taster \\
\hline p.(Leu107CfsX5) & NA & NA & Disease-causing \\
p.(Thr375Ala) & $\operatorname{PrD~(0.997)}$ & Damaging (0.010) & Disease-causing \\
p.(Leu846Pro) & $\operatorname{PrD~(0.995)}$ & Damaging (0.001) & Disease-causing \\
p.(Arg1173Gly) & $\operatorname{PrD(0.999)}$ & Damaging (0.007) & Disease-causing \\
p.(Arg1274Trp) & $\operatorname{PrD~(0.986)}$ & Damaging (0.004) & Disease-causing \\
p.(Thr204Thr) & NA & Tolerated (0.703) & Disease-causing \\
c.(1947 + 10G > A) & NA & NA & Polymorphism \\
\hline
\end{tabular}

PolyPhen-2 score ranges from 0 to 1 , with mutations qualitatively appraised as PrD (probably damaging), PoD (possibly damaging), or B (benign).

MutationTaster analyses the probability that a variant will be disease-causing or is a polymorphism. SIFT scores range from 0 to 1 : the amino acid substitution is predicted as damaging if the score is $\leq 0.05$, and tolerated if the score is $>0.05$. $N A$ not possible to analyze

assessment of NP-C SI score in patients with suspected NP-C allows better targeting of in-depth confirmatory laboratory tests. A reduced number of patients were referred for confirmatory molecular-genetic testing, enabling decreases in both the time and costs associated with diagnosing NP-C.

Considering findings from both study periods together, although the majority of NP-C patients with two NPC1 mutations had elevated levels of both ChT and CCL18/ PARC, two subjects had a clear genetic diagnosis of NP-C but showed raised levels of only one of these biomarkers.

Across the two periods in the current study, we identified a total of six novel $N P C 1$ variants and $17 N P C 1$ mutations that have been described previously [2, 17, $30-36]$. No causal NPC2 mutations were detected. Eight of the patients examined in this study only had a single NPC1 mutation, and it is considered highly likely that all of these patients are true cases of NP-C based on their clinical presentation, filipin staining findings, and plasma ChT, CCL18 and 7-KC values. It is possible that these patients also had a second point mutation that was not detectable with our sequencing and MLPA methodology. Alternatively, these mutations could have resulted from whole-gene deletions, intron sequence variants or mutations in other genes that could modify intra-lysosomal cholesterol transport. Full-gene sequencing is possible using next-generation methods, but this is likely to identify variants that are difficult to interpret.

We observed that patients with homozygous or compound-heterozygous NPC1 mutations, as well as carriers of single NPC1 mutations, had elevated ChT activity and CCL18/PARC concentration, although levels of these biomarkers were higher in NP-C positive patients than in 'NP-C uncertain' individuals. The existence of CHIT1 polymorphisms associated with reduced ChT activity limits the usefulness of ChT as a biomarker in some patients [16]. Nonetheless, the combined analysis of both ChT and CCL18/PARC levels allowed the identification of four additional patients with lysosomal storage diseases: three patients with Niemann-Pick disease type B and one with GD.

The NP-C SI, which was developed in 2012, was applied retrospectively to individuals assessed during Period 1 and prospectively in those assessed during Period 2 in order to 
gain a complete set of diagnostic data. This clinical tool has shown promise in aiding the diagnosis of NP-C in previous studies [6]. However, we observed high prediction scores $(\geq 70)$ in 79 of all included patients $>4$ years old in the current cohort, in which only six patients had a confirmed diagnosis of NP-C. While several patients in this cohort had high NP-C SI scores as well as clinical signs associated with NP-C (VSGP and splenomegaly), genetic analyses did not reveal any NPC1 or NPC2 mutations. Conversely, the NP-C SI cut-off value of 70, recommended as an indicator for additional diagnostic tests, could have led to non-detection of NP-C in two patients who had scores of 60 and 20. The measurement of plasma ChT activity and CCL18/PARC concentration as well as NP-C SI assessments allowed us to confirm a total of eight NP-C diagnoses. In addition, two patients in whom the NP-C SI was not applicable due to their age ( $<4$ years) were detected based on biomarker measurements alone.

It should be borne in mind that elevations in CCL18/ PARC concentration are not exclusively caused by lysosomal disorders. CCL18/PARC is a circulating chemokine that plays a role in injury healing, physiological homing of mononuclear blood cells, and inflammatory responses [37]. Indeed, some studies have indicated that CCL18/ PARC is expressed in atherosclerotic plaques, and represents an independent risk predictor of short-term mortality in patients with acute coronary syndromes [38]. The lack of correlation between CCL18/PARC levels and NP-C SI scores in this study might therefore be due to the presence of other concomitant pathologies such as cardiovascular disease, which are not considered in the NP-C SI.

The filipin test was performed in as many patients as possible in this cohort, and all tested patients with one or two NPC1 mutations showed a classical or variant filipin staining pattern, respectively. However, it is interesting to note that not all carriers of single NPC1 mutations who had a positive filipin result exhibited elevated plasma 7-KC concentrations, whereas all patients with two NPC1 mutations had 7-KC levels above the control cut-off value in our laboratory. It is also notable that family studies addressing ChT/CCL18/7-KC biomarkers in conjunction with the NP-C SI, filipin testing and subsequent gene sequencing led to the identification of a further three NP-C patients, and enabled the validation of two new causal NPC1 variants (p.(Arg1173Gly) and p.(Thr375Ala)).

This investigation has several limitations and strengths that should be taken into account in interpreting the reported findings. Although the inclusion of patients with two typical NP-C symptoms might be considered a bias for having a high NP-C SI score, it should be noted that this study was started in 2010, and the symptoms required for inclusion in this work were specified before the NP-C SI was developed and published in 2012 [6]. Such a bias was therefore not possible. Secondly, we did not sequence all individuals with suspected NP-C who had plasma ChT activity or CCL18/PARC concentration less than the mean control value (plus two SDs). However, we did conduct NP-C gene sequencing and MLPA in all individuals with an NP-C SI $\geq 70$ or who were $<4$ years old. Thirdly, the sequencing techniques that we employed only covered the coding regions of the NPC1 and NPC2 genes and their intron-exon boundaries, and therefore might not have detected regulatory and deep intronic splicing mutations. It is also possible that some novel mutant NP-C gene alleles might not yet have been characterized, and therefore may be present (but go undetected) among 'NP-C uncertain' or even 'NP-C negative' individuals. Finally, the genetic diagnosis of NP-C requires the identification of clearly pathogenic mutations, while many families have 'private' sequence variants that have not yet been reported/published. While in silico protein and splicing prediction tools can be employed to assist in assigning pathogenicity to sequence variants resulting from missense or intronic changes, decisions regarding the pathogenicity of novel, private mutations are generally very difficult to make.

\section{Conclusion}

We conclude that plasma ChT activity and CCL18/PARC concentration measurements in patients with suspected NP-C based on their clinical symptomatology can help pave the way to conducting specific, confirmatory laboratory tests. This approach can be very useful for laboratories that do not have mass spectrometry facilities and therefore they cannot use other NP-C biomarkers such lyso-sphingomyelin-509 or bile acids. Patients with a plasma ChT activity and/or plasma CCL18/PARC concentration greater than two SDs beyond control values and an NP-C SI score $\geq 70$ should undergo NPC1 and $N P C 2$ gene sequencing as standard. In addition, identification of two pathogenic NP-C gene mutations that segregate in the family should be considered sufficient for at least an initial diagnosis.

\section{Additional files}

Additional file 1. Patient questionnaire.

Additional file 2. Filipin staining methodology.

\footnotetext{
Abbreviations

7-KC: 7-ketocholesterol; CHIT1: c.1049_1072dup24 polymorphism of the ChT gene; CCL18/PARC: chemokine (C-C motif) ligand 18/pulmonary and activation-regulated chemokine; ChT: chitotriosidase; ELISA: enzyme-linked
} 
immunosorbent assay; ERT: enzyme replacement therapy; EVS: exome variant server; GBA: glucocerebrosidase; GD: Gaucher disease; HGVS: Human Genome Variation Society; MLPA: multiplex ligationdependent probe amplification; NCBI: National Center for Biotechnology Information; LOD: limits of detection; LC-MS/MS: liquid chromatography-tandem mass spectrometry; LDL: lowdensity lipoprotein; MLPA: multiplex ligation-dependent probe amplification; NP-C: Niemann Pick disease type C; NPC1/NPC2: NP-C disease-causing genes 1 and 2; NP-C SI: NP-C suspicion index; SD: standard deviation; SMDP1: acid sphingomyelinase gene; VSGP: vertical supranuclear gaze palsy.

\section{Authors' contributions}

Study conception and design: MP. Drafting the article or revising it critically for important intellectual content: ICO, PI, CD, MP. Acquisition, analysis or interpretation of data: ICO, PI, JJC, MM, MJP, PM, VRS. Obtained funding: MP, CD. The members of the Spanish NPC Group (see Acknowledgements) provided patient samples and/or clinical data. All authors read and approved the final manuscript.

\section{Author details}

${ }^{1}$ Department of Biochemistry and Molecular and Cellular Biology, Faculty of Science, University of Zaragoza, C. Pedro Cerbuna 12, 50009 Saragossa, Spain. ${ }^{2}$ Instituto de Investigación Sanitaria Aragón (IIS Aragón), Saragossa, Spain. ${ }^{3}$ Centro de Investigación Biomédica en Red (CIBERER), Instituto de Salud Carlos III, Saragossa, Spain. ${ }^{4}$ Spanish Foundation for the Study and Therapy of Gaucher Disease, Saragossa, Spain. ${ }^{5}$ Biochemistry and Molecular Biology Research Centre for Nanomedicine, Vall d'Hebron University Hospital, Barcelona, Spain

\section{Acknowledgements}

Matthew Reilly Ph.D. at InTouch Medical Ltd provided editorial support in the preparation of this manuscript for publication.

The Spanish NPC Group includes all physicians who provided patient samples and clinical data for the study (see Additional file 1 for full listing).

The Spanish NP-C Group: Alejandro Bustamante, Irene Perez Ortega, Pablo Mir Rivera, Alfredo Palomino, Maite Caceres, Silvia Jesus Maestre and Enrique Calderon (Hospital Virgen del Rocio, Sevilla); Juan Jesus Rodriguez Uranga, Juan Bautista Lorite and Ma Dolores Gomez Bustos (Clinica Sagrado Corazón, Sevilla); Jose M. Garcia Moreno (Hospital Virgen de la Macarena, Sevilla); Teresa Bermejo Gonzalez (Instituto Inspalense Pediatria, Sevilla); Alfredo Muñoz (SAS Jerez, Jerez); Manuel Romero Acebal (Hospital Clinico Universitario de Málaga, Málaga); Rocio Calvo Medina, Juliana Serrano Nieto, Esmeralda Nuñez (Hospital Carlos Haya, Málaga); Carlos Sierra (Hospital Materno Infantil, Málaga); Mercedes Gil Campos, Rafael Fernandez de la Puebla, Juan José Ochoa Sepulveda, Eduardo Lopez Laso (Hospital Reina Sofía, Cordoba); Asuncion Maestre (Complejo Hospitalario de Jaen, Jaen); Myriam Ley Martos, Pamela Zafra, Servando Pantoja Rosso, Raul Espinosa Rosso, Ma Jesus Salado Reyes (Hospital Puerta del Mar, Cadiz); Luisa Arrabal Fernandez, Angel Ortega Moreno, Cristobal Carnero Pardo (Virgen de las Nieves Hospital, Granada); Alejandro Martin (Hospital Clinico de Salamanca, Salamanca); Jordi Alom Poveda (Hospital General Universitario de Elche, Alicante); Belén Nacimiento Cantero (Hospital Puerta del Hierro, Madrid); Pedro Garcia Ruiz, Maria Rodrigo (Fundacion Jimenez Diaz, Madrid); Ignacio Posada, Alvaro Sanchez Ferro, Jesús Hernandez Gallego, Alberto Villarejo, Alejandro O. Herrero San Martin (Hospital 12 Octubre, Madrid); Asuncion Garcia Perez (Fundacion Alcorcon, Madrid); Francisco Javier Rodriguez de Rivera, Irene Sanz, Fernando Santos (Universitario la Paz, Madrid); Luis Gutierrez-Solana (Hospital Niño Jesus, Madrid); Carmen Fontan, Susana Cantarero Duque (Hopital de Mostoles, Madrid); Dr. Rafael Martinez Leal (Hospital Villablanca, Reus); Josep Gamez, Esteve Santamaria, Mireia del Toro (Hospital Vall d'Hebron, Barcelona); Tania Delgado, Isabel Lorente (Parc Tauli; Sabadell, Barcelona); Alberto Lleó, Javier Pagonabarraga, Marc Suarez Calvet, Eulalia Turón, Elisenda Moliner, Berta Pascual (Hospital Santa Creu i Sant Pau, Barcelona); Consuelo Almenar (Hospital Benito Menni. Sant Boi de Llobregat, Barcelona); Anna Olivé Torralba, Robert Misericordia Floriach (Hospital, Mare de Déu de la Merce, Barcelona); Nilda Venegas Bernal (Parc Sanitari SJD, Barcelona); Ma Dolores Lopez Villegas; Luis Planellas Giné (Hospital del Mar, Parc de Salut Mar, Barcelona); Benet Nomdedeu, Ma Jose Martí, Ma Teresa Bounjourno Domenech (Hospital Clinic, Barcelona); Jordi Gascón (Hospital Bellvitge, Barcelona); Joan Costa, Carmen Fons, Mercedes Pineda (Hospital San Juan de Dios, Esplugues, Barcelona); Ma Teresa Abellan Vidal, Emili Mira (Parc Salut Mar Sta, Coloma); Juan Pablo Tartari (Mutua Terassa, Terrasa); Roser Castilla Aparici (Psiquiatrico Sagrado
Corazon, Martorell); Antonio Arevalo Sanchez (Hospital Sagrat Cor, Martorell); Marc Boix Codony, Ma Antonia Alberti (Hospital Arnau de Vilanova, Lleida); Gerard Piñol Ripoll (Hospital Provincial Santa Maria, Lleida); Ramon Modol (Hospital San Juan de Dios, Almacelles, Lleida); David Genis (Hospital Dr Josep Trueta, Girona); Rafael Sivera, Juan Fco Vazquez Costa, Irene Martinez, Patricia Smeyers, Tomas Vila, Bonaventura Casanova, Francisco Carlos Perez Miralles, Jaime Dalmau Serra, Juan Fco Vazquez Costa, Isidro Vitoria Miñana (Hospital La Fe, Valencia); Caridad Valero Merino (Hospital Arnau de Vilanova, Valencia); Ribas Garcia, Raquel, Margarita Simo Jorda (Hospital Dr Peset, Valencia); Juana Clavel (Hospital de Castellón, Castellón); Carlos Leiva Santana (Hospital General Universitario de Alicante, Alicante); Jose Luis Capablo, Jose Gazulla (Hospital Universitario Miguel Servet, Zaragoza); Pablo Padilla (Centro Neuropsiquiatrico Nuestra Señora del Carmen, Zaragoza); Carmen Loureiro, Carmen Navarro Fdez-Balbuen (C.H.U. Vigo, Meixoeiro, Vigo); Manuel Arias; $M^{a}$ José Rabuñal (CHU de Santiago, Coruña); Ma Jesus Sobrido (Hospital San Rafael, Coruña); Ma Teresa Cia, Ma Eugenia Yoldi (Hospital Virgen del Camino, Pamplona); Ma Elena Erro, Ariadna Fontes (Complejo Hospitalario Navarra, Pamplona); Idoia Rouco Axpe, Francisco Javier Ezkurida Sasieta, Juan Carlos Gomez Esteban (Hospital de Cruces, Vizcaya); Jose A Suarez Muñoz (Hospital Dr. Negrin, LPGC); Norberto Rodriguez Espinosa (Hospital Universitario Virgen Candelaria, Tenerife); Miguel Angel Hernandez, Ingrid Tejera Martin (Hospital Nuestra Señora Candelaria, Tenerife); Alberto Fuentes Garrido (CSM Otero, Ceuta); Dr. Rafael Aporta (Hospital Univ. Ceuta, Ceuta); Rosario Domingo (Hospital Virgen Arrixaca, Murcia); Ignacio Casado Naranjo (Hospital San Pedro Alcantara, Caceres); Ma Angeles Ruiz Gomez, Guillermo Amer Ferrer (Hospital Son Espases, Palma, Mallorca).

\section{Competing interests}

The authors declare that they have no competing interests. No funding organization played any role in the study design, the collection, analysis and interpretation of data, or in the decision to submit the article for publication.

\section{Availability of data and materials}

The datasets during and/or analysed during the current study are available from the corresponding author on reasonable request.

\section{Ethics approval and consent to participate}

Written informed consent was obtained from all patients or their guardians before they underwent any study-related procedures. The study protocol was approved by the Aragon Experimental Ethical Committee (CEICA) in Spain, and was developed in accordance with the 1975 Helsinki declaration, as revised in 2000.

\section{Funding}

This work was supported by Grants from the Spanish Fondo de Investigaciones Sanitarias. FIS PI12/01219, Fundación para el Estudio y Terapéutica de la Enfermedad de Gaucher (FEETEG), and Centro de Investigación Biomédica en Red de Enfermedades Raras (CIBERER), which is an initiative of the Carlos III Institute of Health (ISCIII).

Received: 2 November 2016 Accepted: 9 February 2017 Published online: 21 February 2017

\section{References}

1. Vanier MT. Niemann-Pick disease type C. Orphanet J Rare Dis. 2010;5:16.

2. Carstea ED, Morris JA, Coleman KG, Loftus SK, Zhang D, Cummings C, et al. Niemann-Pick C1 disease gene: homology to mediators of cholesterol homeostasis. Science. 1997;277:228-31.

3. Naureckiene S, Sleat DE, Lackland H, Fensom A, Vanier MT, Wattiaux R, et al. Identification of HE1 as the second gene of Niemann-Pick C disease. Science. 2000;290:2298-301.

4. Patterson MC, Hendriksz CJ, Walterfang M, Sedel F, Vanier MT, Wijburg F, et al. Recommendations for the diagnosis and management of NiemannPick disease type C: an update. Mol Genet Metab. 2012;106:330-44.

5. Kelly DA, Portmann B, Mowat AP, Sherlock S, Lake BD. Niemann-Pick disease type C: diagnosis and outcome in children, with particular reference to liver disease. J Pediatr. 1993;123:242-7. 
6. Wijburg FA, Sedel F, Pineda M, Hendriksz CJ, Fahey M, Walterfang M, et al. Development of a suspicion index to aid diagnosis of Niemann-Pick disease type C. Neurology. 2012;78:1560-7.

7. Jiang X, Sidhu R, Porter FD, Yanjanin NM, Speak AO, te Vruchte D, et al. A sensitive and specific LC-MS/MS method for rapid diagnosis of NiemannPick C1 disease from human plasma. J Lipid Res. 2011;52:1435-45.

8. McKay K, Gissen P. Genetic and laboratory diagnostic approach in Niemann Pick disease type C. J Neurol. 2014;261(Suppl 2):569-75.

9. Vanier MT, Rodriguez-Lafrasse C, Rousson R, Gazzah N, Juge MC, Pentchev PG, et al. Type C Niemann-Pick disease: spectrum of phenotypic variation in disruption of intracellular LDL-derived cholesterol processing. Biochim Biophys Acta. 1991;1096:328-37.

10. Argoff CE, Comly ME, Blanchette-Mackie J, Kruth HS, Pye HT, Goldin E, et al. Type C Niemann-Pick disease: cellular uncoupling of cholesterol homeostasis is linked to the severity of disruption in the intracellular transport of exogenously derived cholesterol. Biochim Biophys Acta. 1991;1096:319-27.

11. Tangemo C, Weber D, Theiss S, Mengel E, Runz H. Niemann-Pick Type $C$ disease: characterizing lipid levels in patients with variant lysosomal cholesterol storage. J Lipid Res. 2011;52:813-25.

12. Hollak CE, van Weely S S, van Oers MH, Aerts JM. Marked elevation of plasma chitotriosidase activity. A novel hallmark of Gaucher disease. J Clin Invest. 1994;93:1288-92.

13. Guo Y, He W, Boer AM, Wevers RA, de Bruijn AM, Groener JE, et al. Elevated plasma chitotriosidase activity in various lysosomal storage disorders. J Inherit Metab Dis. 1995:18:717-22.

14. Renkema GH, Boot RG, Au FL, Donker-Koopman WE, Strijland A, Muijsers $\mathrm{AO}$, et al. Chitotriosidase, a chitinase, and the 39-kDa human cartilage glycoprotein, a chitin-binding lectin, are homologues of family 18 glycosyl hydrolases secreted by human macrophages. Eur J Biochem/FEBS 1998;251:504-9.

15. Ries M, Schaefer E, Luhrs T, Mani L, Kuhn J, Vanier MT, et al. Critical assessment of chitotriosidase analysis in the rational laboratory diagnosis of children with Gaucher disease and Niemann-Pick disease type A/B and C. J Inherit Metab Dis. 2006;29:647-52.

16. Irun P, Alfonso P, Aznarez S, Giraldo P, Pocovi M. Chitotriosidase variants in patients with Gaucher disease. Implications for diagnosis and therapeutic monitoring. Clin Biochem. 2013;46:1804-7.

17. Yamamoto T, Nanba E, Ninomiya H, Higaki K, Taniguchi M, Zhang H, et al. NPC1 gene mutations in Japanese patients with Niemann-Pick disease type C. Hum Genet. 1999;105:10-6.

18. Schutyser E, Richmond A, Van Damme J. Involvement of CC chemokine ligand 18 (CCL18) in normal and pathological processes. J Leukoc Biol. 2005:78:14-26.

19. Boot RG, Verhoek M, de Fost M, Hollak CE, Maas M, Bleijlevens B, et al. Marked elevation of the chemokine CCL18/PARC in Gaucher disease: a novel surrogate marker for assessing therapeutic intervention. Blood. 2004:103:33-9.

20. Chang KL, Hwu WL, Yeh HY, Lee NC, Chien YH. CCL18 as an alternative marker in Gaucher and Niemann-Pick disease with chitotriosidase deficiency. Blood Cells Mol Dis. 2010;44:38-40.

21. Pineda M, Perez-Poyato MS, O'Callaghan M, Vilaseca MA, Pocovi M, Domingo $R$, et al. Clinical experience with miglustat therapy in pediatric patients with Niemann-Pick disease type C: a case series. Mol Genet Metab. 2010:99:358-66.

22. Lin N, Zhang H, Qiu W, Ye J, Han L, Wang Y, et al. Determination of 7-ketocholesterol in plasma by LC-MS for rapid diagnosis of acid SMasedeficient Niemann-Pick disease. J Lipid Res. 2014;55:338-43.

23. Porter FD, Scherrer DE, Lanier MH, Langmade SJ, Molugu V, Gale SE, et al. Cholesterol oxidation products are sensitive and specific blood-based biomarkers for Niemann-Pick C1 disease. Sci TransI Med. 2010;2:56.

24. Adzhubei IA, Schmidt S, Peshkin L, Ramensky VE, Gerasimova A, Bork P, et al. A method and server for predicting damaging missense mutations. Nat Methods. 2010;7:248-9.
25. Kumar P, Henikoff S, Ng PC. Predicting the effects of coding non-synonymous variants on protein function using the SIFT algorithm. Nat Protoc. 2009:4:1073-81.

26. Schwarz JM, Rodelsperger C, Schuelke M, Seelow D. MutationTaster evaluates disease-causing potential of sequence alterations. Nat Methods. 2010;7:575-6.

27. Brunak S, Engelbrecht J, Knudsen S. Prediction of human mRNA donor and acceptor sites from the DNA sequence. J Mol Biol. 1991;220:49-65.

28. Reese MG, Eeckman FH, Kulp D, Haussler D. Improved splice site detection in Genie. J Comput Biol. 1997;4:311-23.

29. Baila-Rueda L, Cenarro A, Cofán M, Orera I, Barcelo-Batllori S, Pocoví M. Simultaneous determination of oxysterols, phytosterols and cholesterol precursors by high performance liquid chromatography tandem mass spectrometry in human serum. Anal Methods. 2013;5:2249-57.

30. Fernandez-Valero EM, Ballart A, Iturriaga C, Lluch M, Macias J, Vanier MT, et al. Identification of 25 new mutations in 40 unrelated Spanish Niemann-Pick type C patients: genotype-phenotype correlations. Clin Genet. 2005;68:245-54.

31. Millat G, Bailo N, Molinero S, Rodriguez C, Chikh K, Vanier MT. NiemannPick $C$ disease: use of denaturing high performance liquid chromatography for the detection of NPC1 and NPC2 genetic variations and impact on management of patients and families. Mol Genet Metab. 2005;86:220-32.

32. Millat G, Marcais C, Tomasetto C, Chikh K, Fensom AH, Harzer K, et al. Niemann-Pick C1 disease: correlations between NPC1 mutations, levels of NPC1 protein, and phenotypes emphasize the functional significance of the putative sterol-sensing domain and of the cysteine-rich luminal loop. Am J Hum Genet. 2001;68:1373-85.

33. Greer WL, Riddell DC, Murty S, Gillan TL, Girouard GS, Sparrow SM, et al. Linkage disequilibrium mapping of the Nova Scotia variant of NiemannPick disease. Clin Genet. 1999;55:248-55.

34. Park WD, O'Brien JF, Lundquist PA, Kraft DL, Vockley CW, Karnes PS, et al. Identification of 58 novel mutations in Niemann-Pick disease type C: correlation with biochemical phenotype and importance of PTC1-like domains in NPC1. Hum Mutat. 2003;22:313-25.

35. Ribeiro I, Marcao A, Amaral O, Sa Miranda MC, Vanier MT, Millat G. Niemann-Pick type C disease: NPC1 mutations associated with severe and mild cellular cholesterol trafficking alterations. Hum Genet. 2001;109:24-32.

36. Tarugi P, Ballarini G, Bembi B, Battisti C, Palmeri S, Panzani F, et al. Niemann-Pick type C disease: mutations of NPC1 gene and evidence of abnormal expression of some mutant alleles in fibroblasts. J Lipid Res. 2002:43:1908-19.

37. De Sutter J, Struyf S, Van de Veire NR, Philippe J, De Buyzere M, Van Damme J. Cardiovascular determinants and prognostic significance of CC Chemokine Ligand-18 (CCL18/PARC) in patients with stable coronary artery disease. J Mol Cell Cardiol. 2010;49:894-6.

38. De Jager SCA, Bongaerts BWC, Weber M, Kraaijeveld AO, Rousch M, Dimmeler S, et al. Chemokines CCL3/MIP1 a, CCL5/RANTES and CCL18/PARC are independent risk predictors of short-term mortality in patients with acute coronary syndromes. PLoS ONE. 2012;7:e45804.

\section{Submit your next manuscript to BioMed Central and we will help you at every step:}

- We accept pre-submission inquiries

- Our selector tool helps you to find the most relevant journal

- We provide round the clock customer support

- Convenient online submission

- Thorough peer review

- Inclusion in PubMed and all major indexing services

- Maximum visibility for your research

Submit your manuscript at www.biomedcentral.com/submit 